\title{
K-12 Students as Ground Observers of Contrails in Support of Scientific Research
}

\author{
L. H. Chambers (l.h.chambers@larc.nasa.gov) \\ NASA Langley Research Center, MS 420, Hampton, VA 23681-2199 \\ S. W.Moore(s.w.moore@larc.nasa.gov) \\ J. D. Fischer (j.d.fischer@larc.nasa.gov), \\ R. Sepulveda (r.sepulveda@larc.nasa.gov) \\ Science Applications International Corporation, Hampton, VA \\ C. Clark (cclark@macnet.com), \\ Chapman School, Sheridan, OR
}

\begin{abstract}
Scientists are very interested in the formation of contrails, both the type and the coverage. To be detected by a satellite-born instrument, the contrail must be of a certain size, which means that some contrails go undetected. The K-12 education community is assisting with the study of contrails by participating in a network of student observers. To provide a venue for student contrail observations, the GLOBE Contrails protocol was developed as part of the GLOBE Atmospheric Science protocols. The first year of observations has provided a rich resource for researcher.
\end{abstract}

\section{Introduction}

Clouds are the largest variable controlling Earth's atmospheric temperature and climate. Any change in global cloud cover may contribute to long-term changes in Earth's climate. Likewise, any change in Earth's climate may have effects on natural resources. Contrails, especially persistent contrails, represent a human-caused increase in the Earth's cloudiness, and are likely to be affecting climate and ultimately our natural resources. Scientists are most interested in persistent contrails because they form longlasting and sometimes extensive clouds that would not normally have formed in the atmosphere. Persistent contrails can last for hours to days, and spread over thousands of square kilometers, becoming indistinguishable from naturally occurring cirrus clouds. Scientists are concerned about contrails because predicted increases in air-traffic could result in a continued increase in cloud cover. Knowing when and where contrails form is key to determining their contribution to cirrus cloud cover and their effect on the energy balance. Thus, collecting information on short-lived contrails is also of interest.

\section{ENGAGEMENT OF THE K-12 EDUCATION COMMUNITY}

\section{A. Collaboration between student observers and scientists}

Scientists use satellite imagery from research and weather satellites as an automated means for detecting cloud types. Because contrails are often too small to detect by these satellites, ground observations provide additional data for scientific use. Student observers can collaborate with scientists by observing contrails in their area and reporting on the amount and type of contrails present. The results could help atmospheric scientists determine the atmospheric conditions when persistent contrails form and thus help them predict where they will form and possibly assist air traffic managers in planning different flight-paths or altitudes to avoid contrail formation. To provide a venue for student contrail observations, the GLOBE Contrails protocol was developed as part of the GLOBE Atmospheric Science protocols. Along with the S'COOL Program [1], GLOBE is currently the only formal contrail- observing program that we are aware of in the world. The GLOBE program is an international network of student-observers, studying atmosphere, hydrology, soils, land cover, and Earth as a system. In the first year, nearly 75,000 GLOBE student observations of contrails were recorded, constituting a rich resource for researchers.

\section{B. Web site Development}

In support of the K-12 educators and students implementing the GLOBE contrail protocol, scientists and educators have collaborated in development of the Contrail Education website http://asd www.larc.nasa.gov/GLOBE. As part of the GLOBE Scientist's Corner, the Contrail Education Website provides information about the importance of 
student-observations of contrails, along with the science, history, and satellite-imagery related to a study of contrails.

\section{Assisting the Student Observer}

Following the GLOBE cloud protocol, the student observer collects Contrail Type and Contrail Coverage data. To assist student observers with identification of Contrail Type, the Contrail Education website features a "Contrail Gallery" of multiple photographic images, submitted by educators and scientists, of the three types of contrails as specified by the GLOBE protocol, along with variations of the three for enrichment purposes. Also featured is the "GLOBE Contrail ID Chart”, available in English and four other languages, located in the "Resources" section of the website. Pertinent lessons and activities are also provided. To assist K-12 educators with implementing contrail observation as a learning objective within the curriculum, National Standards-based lessons, activities, and additional resources are available on the website.

To assist student observers with estimation of Contrail Coverage, guidelines are provided within the Cloud and Contrails Protocol, accessible to students and educators through the GLOBE [2] website. Lessons and activities targeting cloud coverage are also available within the protocol and on the Contrail Education website. Lessons and activities have been contributed and reviewed by educators from the elementary, middle, and high school levels, as well as by research scientists working with the GLOBE contrail team.

\section{Using Student Observation Data: Initial Analysis}

The temperature and humidity in the atmosphere govern the formation of persistent contrails by jet aircraft exhaust. Student observations of contrails and clouds recorded through the GLOBE program, including the special event, Earth Day 2004 Contrail Count-a-Thon, are helping NASA Langley scientists to determine the quality and consistency of the humidity data used in numerical weather prediction models, with the ultimate goal of improving the prediction of persistent contrail formation. Nearly 1500 GLOBE observations of clouds and contrails across the continental US were compared to relative humidity calculations from Rapid Update Cycle (RUC) and Advanced Regional Prediction Systems (ARPS) model analyses during April 2004 [3].

Figures 1 and 2 show how the relative humidity with respect to ice (RHI) at aircraft cruise levels compares for each model based on contrail observation criteria. Figure 1 shows that the RHI over schools that observed only short-lived contrails averaged 54 and 62 percent for the RUC and ARPS models respectively, while Figure 2 shows the average RHI measured when spreading persistent contrails were observed was 67 and 77 percent.
Fig.1
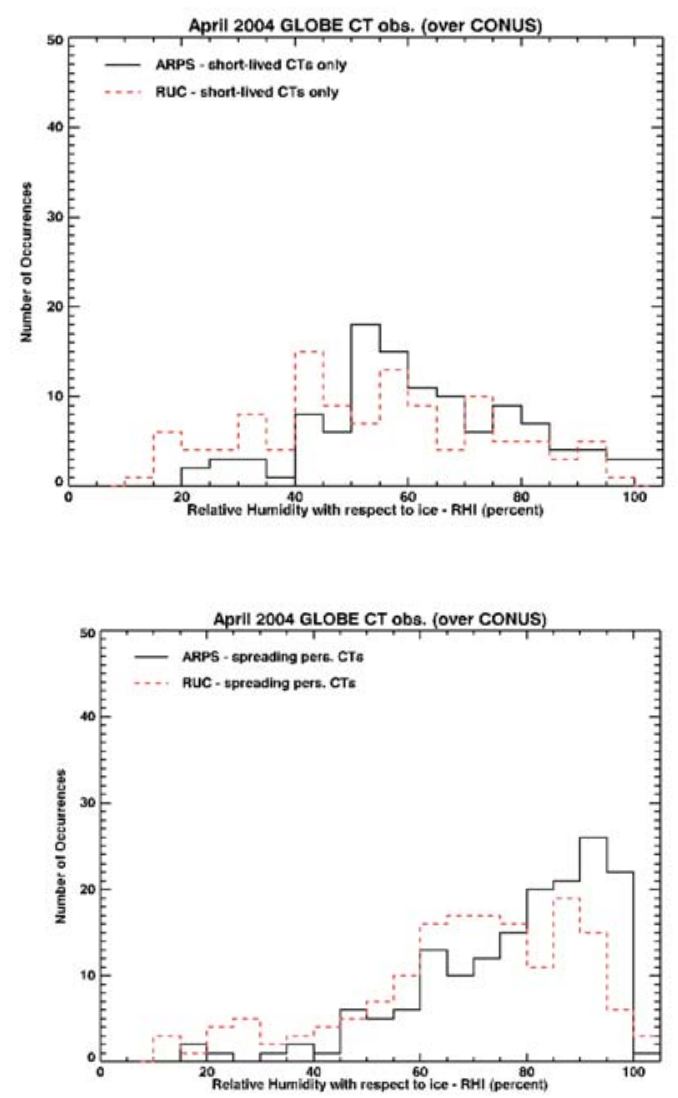

Comparisons like these with GLOBE data will allow atmospheric scientists to improve estimates of the current and future cloud coverage caused by persistent contrails.

\section{FUTURE OPPORTUNITIES}

As student observers continue to contribute data to the GLOBE database, and as more teachers become trained in the GLOBE protocols, the role of NASA Langley as a GLOBE partner is predicted to increase. Future workshops are being planned. Assistance is being provided to local teachers who are implementing the GLOBE Clouds and Contrails protocol. Lessons and activities will be added to the Contrail Education website to support the collaboration between student observers and research scientists. The K12 education community will continue to provide ground observation data to support contrail research, and additional analyses will be performed.

\section{ACKNOWLEDGMENT}


Support for the GLOBE Contrail Protocol was provided by the National Science Foundation and the NASA Earth Science Enterprise.

\section{REFERENCES}

[1] R.Sepulveda, L.H. Chambers, "NASA CERES S'COOL Project: Bridging the gap between research and the classroom”, International Geophysical and Remote Sensing Symposium, Anchorage, AK, September 2004.

[2] GLOBE Program (www.globe.gov)

[3] Chambers, L. and D.Duda, "The GLOBE Contrail Protocol: Initial Analysis of Results”, GLOBE Annual Meeting, Boulder, CO 2004 